\title{
Soil pH determines the alpha diversity but not beta diversity of soil fungal community along altitude in a typical Tibetan forest ecosystem
}

\author{
Jun-Tao Wang • Yuan-Ming Zheng • Hang-Wei Hu • \\ Li-Mei Zhang $\cdot$ Jing Li $\cdot$ Ji-Zheng He
}

Received: 18 December 2014 / Accepted: 15 January 2015 /Published online: 5 February 2015

(C) Springer-Verlag Berlin Heidelberg 2015

\begin{abstract}
Purpose Despite their symbiotic relationship with trees and the vital role as decomposer in forest, soil fungi received limited attention regarding their changes with altitude in forest ecosystems. This study aimed to determine the diversity patterns of soil fungi along an altitudinal gradient on Mt. Shegyla, a typical forest ecosystem on the Tibetan Plateau.

Materials and methods High-throughput barcoded pyrosequencing and quantitative PCR approaches were employed to measure the community composition, diversity, and abundance patterns of soil fungal 18S ribosomal RNA (rRNA) gene in 20 samples collected along the altitudinal gradient of Mt. Shegyla.

Results and discussion Abundant taxa in the fungal community were Agaricomycetes and Leotiomyceta on Mt. Shegyla. Fungal abundance decreased significantly with increasing altitude. Beta diversity of the fungal community, as measured using weighted UniFrac distance, was significantly related to altitude. Significant correlation was observed between altitude and alpha diversity including richness and phylodiversity, but not with evenness. Network analysis revealed that Ceramothyrium and Clavulina were two important hubs in
\end{abstract}

Responsible editor: Zhihong $\mathrm{Xu}$

J.-T. Wang $\cdot$ Y.-M. Zheng $(\bowtie) \cdot$ L.-M. Zhang $\cdot$ J. Li $\cdot$ J.-Z. He State Key Laboratory of Urban and Regional Ecology, Research Center for Eco-Environmental Sciences, Chinese Academy of Sciences, Beijing 100085, China

e-mail: zhengym@rcees.ac.cn

J.-T. Wang

University of Chinese Academy of Sciences, Beijing 100049, China

H.-W. Hu $\cdot$ J.-Z. He $(\bowtie)$

Faculty of Veterinary and Agricultural Sciences, The University of Melbourne, Parkville Campus, Victoria 3010, Australia

e-mail: jzhe@rcees.ac.cn the community, and an uncultured fungal taxon that previously detected in glacier forefront dominated this network. Distance-based linear model identified soil $\mathrm{pH}$ as the dominant driver which significantly related with fungal alpha diversity including richness, phylodiversity, and evenness. However, fungal abundance and the first component of PCoA on weighted UniFrac matrix (beta diversity) did not change significantly with $\mathrm{pH}$.

Conclusions These results provided strong evidence that soil $\mathrm{pH}$ was the dominant driver for structuring altitudinal alpha diversity pattern but not beta diversity pattern or community abundance of soil fungi in this typical forest on the Tibetan Plateau.

Keywords Altitude $\cdot$ Diversity $\cdot$ Forest $\cdot$ Fungi $\cdot$ Soil pH . Tibetan Plateau

\section{Introduction}

The Tibetan Plateau harbours vast areas of alpine meadow and forest (Luo et al. 2002). The uplift of the Tibetan Plateau generated an altitudinal gradient of almost $5,000 \mathrm{~m}$ and thereby inevitably modified the global climate (Spicer et al. 2003). Intensified monsoon from the Indian Ocean brought plentiful precipitation in the southern areas across the latitude, facilitating the formation of typical altitudinal forest belts along the steep environmental gradient (Wang et al. 2014). Forest biomes on the Tibetan Plateau suffered from harsh environmental conditions including strong UV, low temperature and low oxygen content, and the support from the underground communities was especially important in sustaining the biological diversity and ecosystem functions in a scenario of global change ( $\mathrm{Li}$ et al. 2013; Bardgett and van der Putten 2014; 
Shen et al. 2014b). Fungi are recognized as a vital component of the belowground community intimately related with plant communities in a forest (He et al. 2005; Mueller et al. 2014; Peay et al. 2013). Soil fungi play a key role as decomposer to accelerate degradation of soil organic carbon and nitrogen input (McGuire et al. 2010; Schneider et al. 2012), and they also have a symbiotic relationship with aboveground vegetation, which benefits plant with more resistance against extreme circumstances like oligotrophic or arid habitats (Compant et al. 2010).

Altitudinal distribution patterns of biodiversity could be interpreted into alpha diversity and beta diversity. The former was usually characterized by richness, evenness, and phylodiversity. Richness pattern of fungi evaluated through the operational taxonomic unit (OTU) counts across individual community demonstrated a species-area relationship along the altitudinal gradient of the Alps (Pellissier et al. 2014). Phylodiversity and evenness pattern demonstrated the fungal variance across altitudinal forest types in the Andes (Geml et al. 2014). The latter reflects the shifts of community composition triggered the turnover of fungi (beta diversity) along altitude (Geml et al. 2014). A previous study revealed that variation of prokaryotic community composition (beta diversity) among different altitudinal belts was much larger than in the same belt (Wang et al. 2014). Soils from different forest types with discrete edaphic properties in the Amazon basin harbored distinct fungal communities (Peay et al. 2013). A general knowledge on fungal community, abundance, and diversity patterns along an altitudinal gradient is essential to precisely interpret the fungal functions and responses to environmental factors.

Factors driving the fungal diversity pattern could be quite variable under different soil conditions. A previous experiment demonstrated $\mathrm{pH}$ as the most powerful driver in structuring the richness and abundance of fungi in an arable soil (Rousk et al. 2010). The superiority of soil nutrients in structuring fungal community was recognized across land-use types (Lauber et al. 2008). Situation might be more complicated for the interaction of various factors along altitude in spontaneous habitat, since different environmental factors covary with altitude (Sundqvist et al. 2013). The Tibetan Plateau was considered as the third pole with glacier and permafrost (Yang et al. 2013). Its harsh environment developed distinct plant/animal taxa in the Tibetan Plateau from the plain areas (Guo et al. 2011). However, we have limited information on the soil fungal community variance and diversity patterns along altitude.

This study aimed (i) to determine the community variance (beta diversity), abundance, and alpha diversity patterns (including richness, evenness, and phylodiversity) of fungi along the altitudinal gradient and (ii) to evaluate fungal effect of different environmental factors in structuring fungal altitudinal distribution patterns in the forest soils on the Tibetan
Plateau. To achieve these goals, we collected soil samples along a 1,300-m altitudinal gradient on Mount (Mt.) Shegyla, a typical forest ecosystem on the Tibetan Plateau, and performed quantitative PCR (qPCR) and high-throughput barcoded pyrosequencing analyses on the soil fungal community.

\section{Materials and methods}

2.1 Soil sample collection, DNA extraction and physicochemical analyses

The Nyingchi District in the southeast Tibet harbors the largest area of forest on the Tibetan Plateau. Different from other regions, Nyingchi has a wetter climate with mean annual precipitation higher than $650 \mathrm{~mm}$, which facilitates the proliferation of forest. Soil samples were collected in July 2011 on Mt. Shegyla (94 $25^{\prime}-94^{\circ} 45^{\prime}$ E, $29^{\circ} 35^{\prime}-29^{\circ}$ $57^{\prime} \mathrm{N}$ ) characterized by the typical altitudinal vegetation in Nyingchi. The base of Mt. Shegyla is 2,100 m (above sea level) and the peak is $5,300 \mathrm{~m}$. Seven altitudinal sites were identified ranging from 3,351 to $4,477 \mathrm{~m}$ where no obvious anthropogenic disturbance was observed. At each site, three surface soil samples $(0-10 \mathrm{~cm})$ were collected from individually separated $10 \mathrm{~m} \times 10 \mathrm{~m}$ plots by pooling eight soil cores randomly taken from each plot. Stones and plant residues were removed. Fresh samples were transported to the laboratory in a freezer and sieved to $2 \mathrm{~mm}$ before storage at $4{ }^{\circ} \mathrm{C}$. A small portion of each soil sample was freeze-dried and preserved at $-80{ }^{\circ} \mathrm{C}$ before DNA extraction. The aboveground vegetation and the altitude of each plot were also recorded.

DNA was extracted from $0.25 \mathrm{~g}$ of fresh soil samples using a MOBIO Ultraclean Soil DNA Isolation Kit (MOBIO laboratories, Carlsbad, CA, USA) following the manufacturer's instructions as previously described (Wang et al. 2014). DNA extracts were qualified using a NanoDrop ${ }^{\circledR}$ ND-2000c UV-Vis spectrophotometer (Thermo Fisher Scientific, Wilmington, USA), and tenfold dilutions were used in the downstream molecular analyses. Soil moisture content was determined by oven-drying fresh soil samples at $105{ }^{\circ} \mathrm{C}$ for $12 \mathrm{~h}$. Soil $\mathrm{pH}$ was measured using a soil to water ratio of $1: 2.5$. Soil organic carbon (SOC) was determined using the $\mathrm{K}_{2} \mathrm{Cr}_{2} \mathrm{O}_{7}$ oxidation method, and total nitrogen (TN) was determined using a Vario EL III analyzer (Elementar Analysensysteme GmbH, Hanau, Germany). Soil clay content $(<0.002 \mathrm{~mm})$ was determined using a Mastersizer 2000 Laser Diffraction Particle Analyzer (Malvern Instruments Ltd., Malvern, UK). Soil cation exchange capacity (CEC) was measured with the Kjeldahl method (Kitsopoulos 1999). Detailed edaphic properties of the soil samples are listed in Table 1. 
Table 1 Site information and edaphic properties of the soil samples from Mt. Shegyla

\begin{tabular}{llllllllll}
\hline Site ID & Altitude $(\mathrm{m})$ & MAT $\left({ }^{\circ} \mathrm{C}\right)$ & Moisture $(\%)$ & Clay $(\%)$ & $\mathrm{pH}$ & $\mathrm{TN}(\%)$ & SOC $(\%)$ & $\mathrm{CEC}\left(\mathrm{cmol} \mathrm{kg}^{-1}\right)$ & Vegetation $($ dominant species) \\
\hline Site 1 & 3351 & 7.7 & $27.5 \pm 4.3$ & $10.4 \pm 2.7$ & $5.81 \pm 0.15$ & $0.28 \pm 0.05$ & $3.79 \pm 0.69$ & $18.9 \pm 2.7$ & Quercus semecarpifolia, Abies \\
Site 2 & 3882 & 3.7 & $30.1 \pm 4.1$ & $28.9 \pm 0.8$ & $4.94 \pm 0.14$ & $0.42 \pm 0.07$ & $6.23 \pm 0.34$ & $33.4 \pm 3.5$ & Aibes georgei var. smithii \\
Site 3 & 3895 & 4.8 & $30.2 \pm 1.7$ & $22.0 \pm 2.6$ & $5.14 \pm 0.12$ & $0.30 \pm 0.01$ & $5.14 \pm 0.06$ & $25.4 \pm 0.9$ & Quercus semecarpifolia, Abies \\
Site 4 & 3915 & 3.7 & $23.2 \pm 4.1$ & $23.4 \pm 2.3$ & $4.86 \pm 0.18$ & $0.35 \pm 0.03$ & $5.94 \pm 0.27$ & $25.7 \pm 2.9$ & Aibes georgei var. smithii \\
Site 5 & 4042 & 3.9 & $38.2 \pm 1.3$ & $23.9 \pm 2.5$ & $4.83 \pm 0.19$ & $0.40 \pm 0.08$ & $5.41 \pm 0.51$ & $28.5 \pm 2.2$ & Aibes georgei var. smithii \\
Site 6 & 4299 & 1.6 & $40.0 \pm 3.2$ & $26.4 \pm 2.2$ & $5.06 \pm 0.11$ & $0.42 \pm 0.07$ & $6.16 \pm 0.37$ & $29.0 \pm 4.4$ & Quercus semecarpifolia, Abies \\
Site 7 & 4477 & 1.4 & $33.1 \pm 4.9$ & $21.1 \pm 1.9$ & $5.19 \pm 0.06$ & $0.34 \pm 0.06$ & $5.54 \pm 0.23$ & $18.3 \pm 4.4$ & Rhododendron, Sabina saltuaria \\
\hline
\end{tabular}

2.2 Quantitative PCR analyses and barcoded pyrosequencing of fungal communities

Quantitative PCR assays were performed targeting the $18 \mathrm{~S}$ ribosomal RNA (rRNA) genes on a BIO-RAD IQ5 thermal cycler (Bio-Rad Laboratories, Inc., CA, USA) to determine the fungal abundance. Primer pair NS1 (GTAGTCATATGC TTGTCTC) and FUN (ATTCCCCGTTACCCGTTG) was used in the quantification of 18S rRNA genes (May et al. 2001). The $25-\mu \mathrm{l}$ PCR reaction mixture contained $12.5 \mu \mathrm{l}$ Premix Ex Taq ${ }^{\mathrm{TM}}$ (Takara Biotechnology, Dalian, China), $1 \mu \mathrm{l}$ DNA template, $2.5 \mu \mathrm{l}$ of each primer $(10 \mu \mathrm{M})$, and $6.5 \mu \mathrm{l}$ sterilized $\mathrm{H}_{2} \mathrm{O}$.

The primer pair EF4 (GGAAGGGRTGTATTTATTAG) and FUN5 (GTAAAAGTCCTGGTTCCCC) targeting 18S rRNA gene was used in the barcoded pyrosequencing (Smit et al. 1999). Primer A-Key (CGTATCGCCTCCCTCGCGCC ATCAG) and Primer B-Key (CTATGCGCCTTGCCAGCC CGCTCAG) were respectively linked to the $5^{\prime}$ end of the forward and reverse primer as the adaptor. Between the forward primer and the Primer A-Key, a 10-bp MID sequence was added for barcode identification. The $25-\mu \mathrm{l}$ PCR reaction system contained $12.5 \mu$ l Premix Ex Taq ${ }^{\mathrm{TM}}$ Version 2.0 (Takara Biotechnology), $2 \mu \mathrm{l}$ DNA template (1-10 ng), $1 \mu \mathrm{l}$ of each primer $(10 \mu \mathrm{M})$, and $8.5 \mu$ sterilized $\mathrm{H}_{2} \mathrm{O}$. The resultant PCR amplicons were purified with a Wizard SV Gel and PCR Clean-Up System (Progema Corporation, Madison, USA) following the manufacturer's instructions. The purified amplicons were mixed equimolarly in a 2-ml tube and sequenced on a 454 GS FLX Titanium pyrosequencer (Roche 454 Life Sciences, Branford, USA).

\subsection{Pyrosequencing data processing}

Processing of the raw sequences obtained through highthroughput barcoded pyrosequencing was performed using the Quantitative Insights into Microbial Ecology (QIIME) pipeline (Caporaso et al. 2010b). Reads with quality scores lower than 20, ambiguous bases and improper primers were discarded before clustering. Chimeras were eliminated by performing the identify_chimeric_seqs.py and filter_fasta.py scripts within QIIME. The resultant high-quality sequences were then clustered into operational taxonomic units (OTUs) at $97 \%$ similarity using UPARSE (Edgar 2013). Taxonomic classification of representative sequences from individual OTU was performed by BLASTing against the QIIME-compatible versions of the Silva database (Pruesse et al. 2007). PyNAST was chosen as the alignment algorithm against the Silva database (Caporaso et al. 2010a). Fungal community composition was described by the mean relative abundance of sequences assigned to different taxa.

Before diversity calculation, a resampling procedure was employed at a depth of 285 sequences per sample to eliminate the bias on diversity comparison as caused by unevenly sequencing. Fungal richness was demonstrated using the OTU counts at the $97 \%$ similarity, and Faith's phylodiversity index was calculated to compare the phylodiversity. Evenness of the fungal community was evaluated using Simpson's evenness index. The weighted UniFrac matrix was calculated as the beta diversity metrics on community variance along altitude (Lozupone et al. 2007).

\subsection{Statistical analysis}

Spearman's correlation analysis was performed to examine the relationships among altitude, edaphic factors, and mean annual temperature (MAT) (Hijmans et al. 2005). Nonmetric multidimensional scaling (NMDS) analysis based on the weighted UniFrac distances matrix was performed to uncover the variance of fungal communities along altitude using the $n m d s . p y$ script in QIIME. Linear and quadratic fittings were performed in SPSS 18.0 to explore the altitudinal patterns of fungal abundance and diversity, and the significance was examined through analysis of variance (ANOVA). A distancebased multivariate linear model (DistLM) was performed to evaluate the effects of different environmental factors on fungal distribution pattern (Anderson 2001) using the Vegan package (Oksanen et al. 2007) in $\mathrm{R}$ ( $\mathrm{R}$ Core Team. 2013).

A network analysis based on the $97 \%$ OTU identity was performed in CoNet (Faust et al. 2012) to explore the linkage among different fungal taxa. A filtering procedure was performed in advance to exclude OTUs with less than 10 
sequences or three occurrences to avoid spurious correlations. Correlation scores were calculated using Spearman correlation, Pearson correlation, Bray-Curtis dissimilarity, mutual information, and Kullback-Leibler dissimilarity, and only those that reached significant level $(P<0.05)$ by at least three methods were considered to be effective. The importance of certain taxon in the network was evaluated using eigenvector centrality (EC) based on the nod's connections with other nods in Gephi (Bastian et al. 2009). High degree nod within module was considered as a module hub that got higher EC score than other nods in the module, while connector of module hub was considered as the most important networks hub with the highest EC score (Deng et al. 2012). Calculation on topological properties and polish of the network were also performed in the Gephi.

\section{Results}

3.1 Variations of environmental factors along the altitudinal gradient

The correlations among altitude, MAT, and edaphic properties were examined using Spearman's correlation matrix. The MAT showed a significant decreasing trend with the increasing altitude $\left(r^{2}=0.82, P<0.001\right)$. No significant relationship could be observed between altitude and any edaphic factors. It was notable that soil $\mathrm{pH}$, varying from 4.01 to 6.03 , exhibited a concave pattern while other factors exhibited unimodal distributions with increasing altitude. Significant correlations were found among nearly all the edaphic factors, especially that soil $\mathrm{pH}$ exhibited significantly negative correlations with clay content $\left(r^{2}=0.48, P<0.05\right), \mathrm{TN}\left(r^{2}=0.48, P<0.05\right)$, SOC $\left(r^{2}=0.65, P<0.01\right)$, and CEC $\left(r^{2}=0.67, P<0.01\right)$, while the latter four factors exhibited significantly positive correlations with each other.

3.2 Fungal community composition, variations of abundance, and diversity along the altitudinal gradient

For fungal community analysis along the altitudinal gradient, we obtained 152,991 high-quality $18 \mathrm{~S}$ rRNA gene sequences through the high-throughput barcoded pyrosequencing to the 20 soil samples and 7,649 sequences on average were assigned to each sample. Community composition was visualized using the mean relative abundance of each taxon in all the samples (Fig. 1). Agaricomycetes (52\%) was the most abundant phylum in the fungal community, followed by Leotiomyceta (29\%), and uncultured soil fungi (9\%). A small proportion of Glomeromycota (2\%) and Chytridiomycota (1\%) were also detected. Quantitative PCR assays revealed that fungal $18 \mathrm{~S}$ rRNA gene abundance on Mt. Shegyla ranged from $4.0 \times 10^{7}$ to $2.0 \times 10^{9}$ copies $\mathrm{g}^{-1}$ soil.

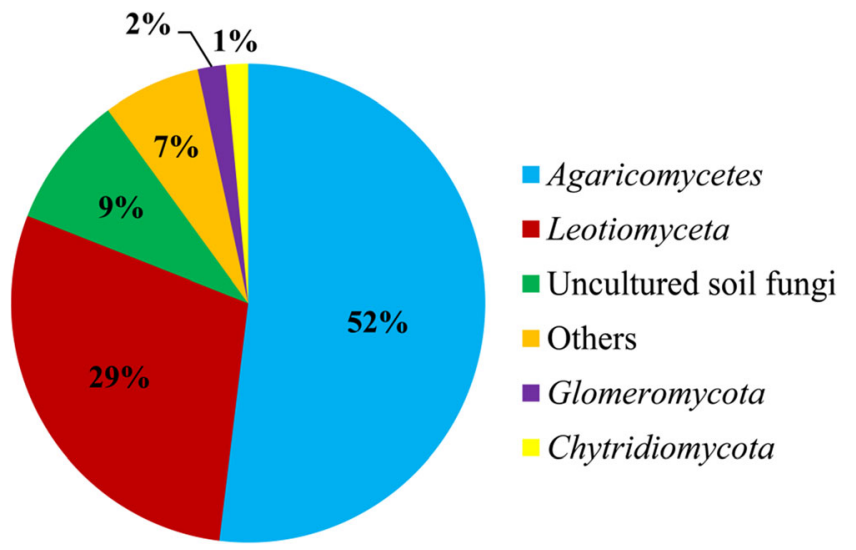

Fig. 1 Fungal community composition in forest soils on Mt. Shegyla. The relative abundance of each taxon was represented using its mean value in all the samples. Taxa were arranged in the pie chart in a decreasing order of sequence counts

Using the weighted UniFrac distance, a nonmetric multidimensional scaling (NMDS) analysis was performed to illustrate the fungal community variance (beta diversity) along the altitude (Fig. 2). Communities from the middle altitudinal sites tended to cluster together, while those from high and low altitudinal sites were more similar to each other. This finding was further corroborated by Mantel test through which a significant relationship was detected between altitude and the weighted UniFrac matrix of the fungal communities (Spearman $r=0.34, P<0.01)$. Fungal abundance and alpha diversity (including richness, evenness, and phylodiversity) patterns along the altitude were also characterized (Table 2). Abundance $(P<0.05)$ and phylodiversity $(P<0.05)$ decreased significantly with the increasing altitude, while richness was lowest at middle elevations $(P<0.05)$. No significant pattern was observed on fungal evenness.

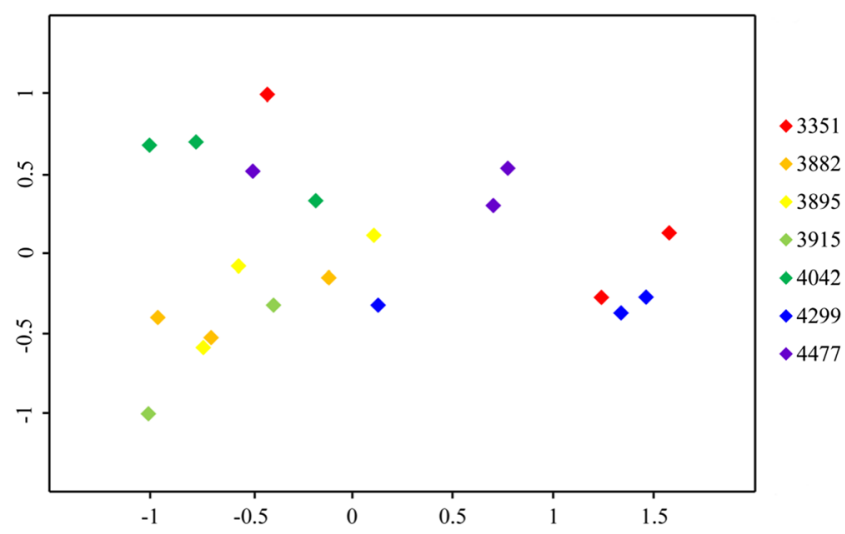

Fig. 2 Nonmetric multidimensional scaling (NMDS) analysis (Stress $=0.05$ ) using the weighted UniFrac distances showing the fungal community variance along the altitudinal gradient on Mt. Shegyla. Colors of the diamonds indicated the altitudinal sites of the samples 
Table 2 Altitudinal distribution patterns of soil fungal abundance and diversity on Mt. Shegyla

\begin{tabular}{lllc}
\hline & \multicolumn{2}{c}{ Altitude } & Pattern \\
\cline { 2 - 3 } & Linear & Quadratic & \\
\hline Abundance & $0.176^{*}$ & 0.137 & У \\
Richness & 0.089 & $0.332^{*}$ & V \\
Evenness & 0.006 & 0.213 & - \\
Phylodiversity & $0.158^{*}$ & 0.203 & У \\
\hline
\end{tabular}

Note: $\$ indicates a decreasing pattern with increasing altitude, while $\bigvee$ indicates concave pattern with increasing altitude. - indicates no significant pattern. The abundance was log transformed. Both linear and quadratic fittings were performed, and analysis of variance (ANOVA) was used to test the model significance. Significance level $P<0.05^{*}$

\subsection{Network analysis of soil fungal communities}

The relationships among different fungal taxa were explored through construction of an OTU network. This network revealed 37 significant correlations (edges) of 28 OTUs (nods) (Fig. 3). Short average path length (1.83) and network diameter (4) showed that the network of different taxa in the fungal community held a characteristic of small world. Notably, by calculating the eigenvector centrality (EC), an index that measures the importance of nod in a certain network, several important taxa were identified in the soils. Cyanodermella (OTU_51, EC 0.24), Ceramothyrium (OTU_156, EC 0.58), Gibberella (OTU_16, EC 0.45) in Leotiomyceta, and Clavulina (OTU_90, EC 0.48) in Agaricomycetes were important nods closely related to the other OTUs. More importantly, an uncultured soil fungi OTU_160 (EC 1.0) was identified as a vital hub closely related to OTU_51 and OTU_90.
3.4 Effect of environmental factors in structuring fungal abundance, alpha and beta diversity patterns along the altitude

A distance-based multivariate linear model (DistLM) was used to distinguish the effects of edaphic properties (including soil $\mathrm{pH}, \mathrm{SOC}, \mathrm{TN}$, moisture, CEC, and clay content), mean annual temperature (MAT), and vegetation in shaping the fungal abundance, alpha and beta diversity pattern along the altitudinal gradient (Table 3). Although soil pH (11\%) and moisture $(10 \%)$ explained the largest part of the community composition variance as measured by the weighted UniFrac matrix, neither of them had a statistically significant effect on fungal beta diversity along altitude. The abundance pattern was neither significantly influenced by any factors examined. However, alpha diversity pattern was closely related with environmental factors, for example, fungal richness was significantly affected merely by soil $\mathrm{pH}(P<0.01)$, and evenness and phylodiversity were dominated by both soil $\mathrm{pH}(P<0.001$, $P<0.05)$ and MAT $(P<0.01, P<0.05)$, respectively.

3.5 Fungal abundance, alpha and beta diversity patterns along the soil $\mathrm{pH}$ gradient

To further investigate the effect of soil $\mathrm{pH}$ on fungal distribution, beta diversity, abundance, and alpha diversity were regressed against soil $\mathrm{pH}$ by the quadratic function (Fig. 4). The first axis of a principal coordinate analysis ( $\mathrm{PCoA})$ using the weighted UniFrac matrix, which explained $44.0 \%$ of the community variance, was not significantly related with soil $\mathrm{pH}\left(r^{2}=0.09, P>0.05\right)$. No significant relationship was detected between fungal abundance and soil $\mathrm{pH}\left(r^{2}=0.12\right.$, $P>0.05$ ). Fungal alpha diversity exhibited strong correlation
Fig. 3 Network analysis on the fungal communities in the soils on Mt. Shegyla. Nod size indicates the eigenvector centrality score, larger nods means more importance in the network. Agaricomycetes, Leotiomyceta, Orbiliomycetes and uncultured soil fungi were colored with red, blue, light cyan, and green, respectively. Other taxa were colored with grey

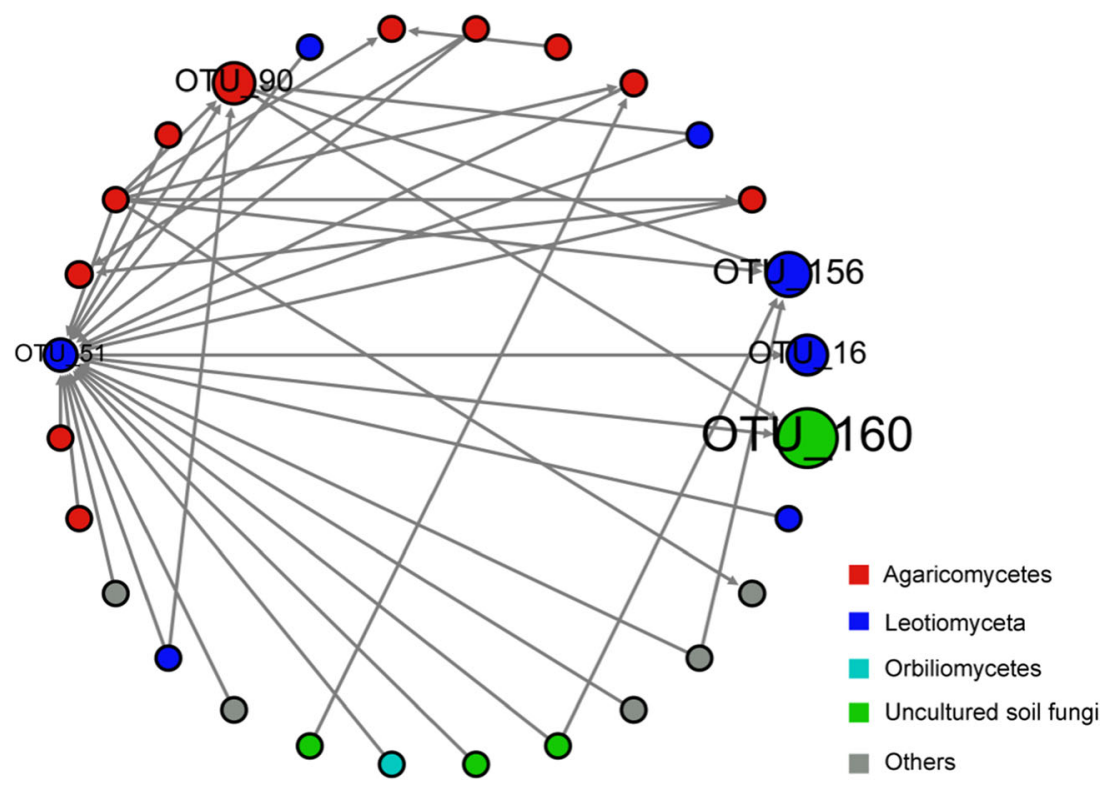


Table 3 Distance-based multivariate linear model (DistLM) revealed the effect of different environment factors on soil fungal community, abundance and diversity on Mt. Shegyla

\begin{tabular}{|c|c|c|c|c|c|}
\hline & \multirow[t]{2}{*}{ Community } & \multirow[t]{2}{*}{ Abundance } & \multicolumn{3}{|l|}{ Diversity } \\
\hline & & & Richness & Evenness & Phylodiversity \\
\hline $\mathrm{pH}$ & 0.11 & 0.12 & $0.25 * *$ & $0.25^{* * *}$ & $0.12^{*}$ \\
\hline MAT & 0.06 & 0.11 & 0.11 & $0.15^{*}$ & $0.14 *$ \\
\hline Moisture & 0.10 & 0.05 & 0.04 & 0.04 & 0.04 \\
\hline $\mathrm{SOC}$ & 0.09 & 0.12 & 0.02 & 0.03 & 0.01 \\
\hline $\mathrm{TN}$ & 0.05 & 0.01 & 0.00 & 0.01 & 0.04 \\
\hline Clay & 0.05 & 0.15 & 0.06 & 0.07 & 0.08 \\
\hline $\mathrm{CEC}$ & 0.02 & 0.03 & 0.03 & 0.05 & 0.11 \\
\hline Vegetation & 0.07 & 0.01 & 0.09 & 0.07 & 0.07 \\
\hline
\end{tabular}

MAT mean annual temperature, SOC soil organic carbon, $T N$ total nitrogen, $C E C$ cation exchange capacity

$* P<0.05 ; * * P<0.01 ; * * * P<0.001$

with soil $\mathrm{pH}$. A significant increasing trend of richness was observed with increasing $\mathrm{pH}\left(r^{2}=0.63, P<0.001\right)$, while an opposite trend was detected as for the evenness $\left(r^{2}=0.65\right.$, $P<0.001)$. Fungal phylodiversity showed a significant increasing trend with the increasing soil $\mathrm{pH}\left(r^{2}=0.22, P<0.05\right)$.

\section{Discussion}

4.1 Characteristics of fungal community in typical forest soils on the Tibetan Plateau

In this study, fungal community composition in the Tibetan forest soils was determined by performing a high-throughput barcoded pyrosequencing on fungal $18 \mathrm{~S}$ rRNA gene. Our results revealed that the fungal community was mostly dominated by Agaricomycetes (Basidiomycota) and Leotiomyceta (Ascomycota), while the relative abundance of Chytridiomycota was quite low. This finding is in good agreement with a previous investigation on fungal communities in the Tibetan Plateau meadow soils (Zhang et al. 2013) where Basidiomycota and Ascomycota were also found to be abundant. However, another study using the clone library approach reported that the fungal communities in the high-elevation soils at the Rocky, Andes, and Himalayan mountains were dominated by Chytridiomycota (Freeman et al. 2009). It should be noted that the latter investigation was carried out on soils of the unvegetated habitat. Because the fungal community was closely related with vegetation (Mueller et al. 2014), the aboveground plant community could be an important
Fig. 4 Effect of $\mathrm{pH}$ in structuring the abundance (a), beta diversity (b), richness (c), evenness (d), and phylodiversity (e) patterns of fungi in the soils on Mt. Shegyla. Beta diversity of fungal community was characterized by the first component of PCoA on weighted UniFrac distance. Quadratic fittings were performed between soil $\mathrm{pH}$ and the indices mentioned
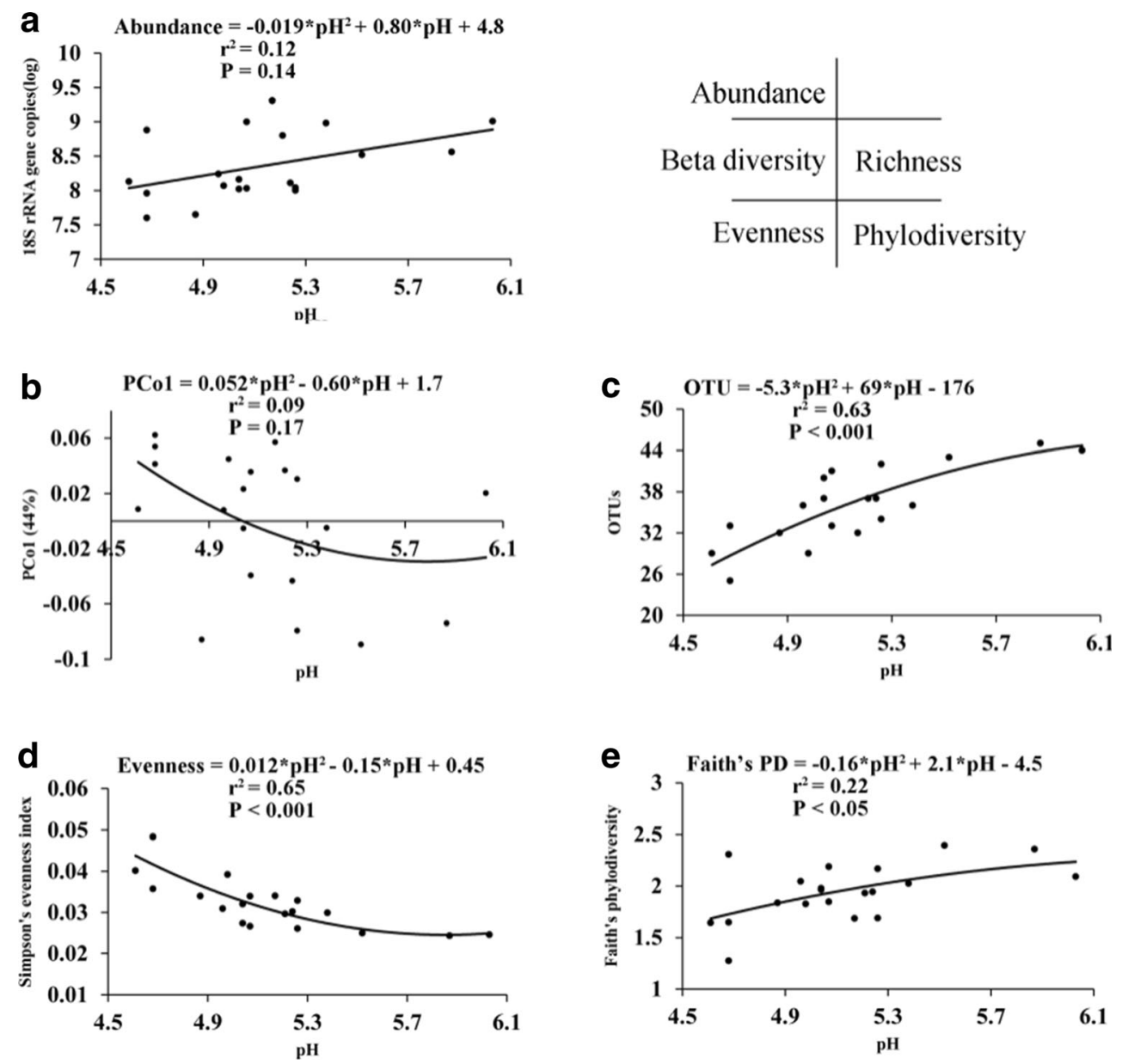
factor that determines fungal community composition. Taxonomic compositions of fungal community in the Tibetan forest soil were also different from that in the Amazon rainforest soils dominated by Sordariomycetes and Saccharomycetes (Peay et al. 2013).

Detailed information on the characteristics of the fungal community in the Tibetan forest soils was obtained through network analysis, a systems biology approach that explored the relationships of different taxa using the high-throughput sequencing data (Raes and Bork 2008). Topologically, the high degree of Cyanodermella (OTU_51) and Clavulina (OTU_90) indicated that they were frequently related to other taxa. A central hub OTU_160 linked Cyanodermella (OTU_51) and Clavulina (OTU_90) was identified through the network analysis. The representative sequence of OTU 160 detected in the Tibetan forest soil was BLASTed to be highly similar to another sequence from a primary successional glacier forefront in the North Cascade Mountains (Jumpponen 2007). This finding suggested that the habitat for fungi in the Tibetan forest soil was, to some extent, similar to that in primary deglaciated environment and highlighted the importance of belowground fungal communities in sustaining aboveground ecosystem under the harsh conditions.

\subsection{Drivers of the fungal diversity patterns}

along the altitudinal gradient

Global patterns of biodiversity were considered to be determined by both stochastic and determinative processes (Martiny et al. 2006). In the case of altitudinal biodiversity patterns, these two processes were further interpreted as a mid-domain effect and an altitudinal environmental gradient, respectively (Colwell and Lees 2000; Sundqvist et al. 2013). Since no unimodal pattern was observed in our research, the altitudinal fungal distribution on Mt. Shegyla could be mostly due to the determinative process (Zhang et al. 2009). Decreased temperature with increasing altitude was the most significant environmental gradient. The temperature gradient largely limited the plant/animal distribution at high elevations through controlling the physiological activity (Hodkinson 2005). Effect of temperature on the altitudinal distribution of microbes was also observed (Bahram et al. 2012; Wang et al. 2012). The MAT did exhibit a significant effect on evenness and phylodiversity patterns of fungi in our research. However, the concave richness pattern emphasized that the spatial heterogeneity of the soils was strongly determinative in structuring altitudinal distribution patterns of the soil microbes (Lauber et al. 2008).

Among all the edaphic factors examined, soil $\mathrm{pH}$ was found to be the most dominant driver that structured fungal richness, phylodiversity, and evenness. The finding that a lower soil $\mathrm{pH}$ supported fewer fungal taxa was quite similar to that from a pH controlled experiment (Rousk et al. 2010). A pH- driven diversity pattern was also detected on soil ammonia oxidizers and eukaryotes (Hu et al. 2013; Shen et al. 2014a). Evenness was another important diversity index that microbiologist should take into account when studying altitudinal biodiversity. In our research, we observed a significant effect of soil $\mathrm{pH}$ on fungal evenness along the altitudinal gradient on Mt. Shegyla. Higher evenness of communities in lower $\mathrm{pH}$ habitat indicated that different taxa were relatively equal in quantity. However, lower $\mathrm{pH}$ was stressful for most taxa, which supported the maintenance but not the prosperity of the community, for instance, acidic mine drainages accommodated much fewer microbial taxa than normal habitat (Kuang et al. 2013). With the increasing $\mathrm{pH}$, some endurable taxa became abundant and the balance of taxa quantity in previous habitat was broken, resulting in the decrease of the community evenness.

Although soil $\mathrm{pH}$ explained more community variance (beta diversity) than other factors like vegetation or nutrients (Table 2), it was still much weaker to identify $\mathrm{pH}$ as a determinant on fungal beta diversity. Contrastingly, $40-50 \%$ of prokaryotic community variance could be explained by soil pH in our previous study (Wang et al. 2014), indicating that fungi was less affected by soil $\mathrm{pH}$ compared with prokaryotes. This finding could be supported by previous $\mathrm{pH}$-manipulated field experiment, in which soil $\mathrm{pH}$ was found to exert much weaker influence on fungal community than on bacterial community (Rousk et al. 2010).

\section{Conclusions}

In conclusion, by performing qPCR and barcode pyrosequencing analyses on the fungal communities of soils from Mt. Shegyla, soil $\mathrm{pH}$ was found to be a more important driver than other edaphic factors that determined the alpha diversity (including richness, evenness, and phylodiversity) pattern, rather than the beta diversity or abundance pattern of soil fungi along the altitudinal gradient in this typical Tibetan forest ecosystem. This finding strengthened our knowledge on belowground biodiversity in sustaining aboveground community and would better help interpret the biodiversity change of the Tibetan Plateau under future environmental scenarios.

Acknowledgments This work was financially supported by grants from National Science Foundation of China (41230857, 51221892), MOST (2013CB956300) and STSN-21-02. We appreciated Drs. Mu Wang and Xi Zha from Agricultural and Animal Husbandry College of Tibet for their assistance in soil sampling.

\section{References}

Anderson MJ (2001) A new method for non-parametric multivariate analysis of variance. Austral Ecol 26:32-46 
Bahram M, Polme S, Kõljalg U, Zarre S, Tedersoo L (2012) Regional and local patterns of ectomycorrhizal fungal diversity and community structure along an altitudinal gradient in the Hyrcanian forests of northern Iran. New Phytol 193:465-473

Bardgett RD, van der Putten WH (2014) Belowground biodiversity and ecosystem functioning. Nature 515:505-511

Bastian M, Heymann S, Jacomy M (2009) Gephi: an open source software for exploring and manipulating networks. In International AAAI conference on weblogs and social media: San Jose, California

Caporaso JG, Bittinger K, Bushman FD, DeSantis TZ, Andersen GL, Knight R (2010a) PyNAST: a flexible tool for aligning sequences to a template alignment. Bioinformatics 26:266-267

Caporaso JG, Kuczynksi J, Stombaugh J, Bittinger K, Bushman FD et al (2010b) QIIME allows analysis of high-throughput community sequencing data. Nat Methods 7:335-336

Colwell RK, Lees DC (2000) The mid-domain effect: geometric constraints on the geography of species richness. Trends Ecol Evol 15:70-76

Compant S, Van Der Heijden MGA, Sessitsch A (2010) Climate change effects on beneficial plant-microorganism interactions. FEMS Microbiol Ecol 73:197-214

R Core Team (2013) R: A language and environment for statistical computing. R Foundation for Statistical Computing, Vienna, Australia. http://www.R-project.org

Deng Y, Jiang Y-H, Yang Y, He Z, Luo F, Zhou J (2012) Molecular ecological network analyses. BMC Bioinformatics 13:113

Edgar RC (2013) UPARSE: highly accurate OTU sequences from microbial amplicon reads. Nat Methods 10:996-998

Faust K, Sathirapongsasuti JF, Izard J, Segata N, Gevers D, Raes J, Huttenhower C (2012) Microbial co-occurrence relationships in the human microbiome. PLoS Comput Biol 8:e1002606

Freeman K, Martin AP, Karki D, Lynch RC, Mitter MS et al (2009) Evidence that chytrids dominate fungal communities in highelevation soils. Proc Natl Acad Sci U S A 106:18315-18320

Geml J, Pastor N, Fernandez L, Pacheco S, Semenova TA et al (2014) Large-scale fungal diversity assessment in the Andean Yungas forests reveals strong community turnover among forest types along an altitudinal gradient. Mol Ecol 23:2452-2472

Guo P, Liu Q, Li C, Chen X, Jiang K, Wang YZ, Malhotra A (2011) Molecular phylogeography of Jerdon's pitviper (Protobothrops jerdonii): importance of the uplift of the Tibetan plateau. J Biogeogr 38:2326-2336

He JZ, Xu ZH, Hughes J (2005) Analyses of soil fungal communities in adjacent natural forest and hoop pine plantation ecosystems of subtropical Australia using molecular approaches based on 18S rRNA genes. FEMS Microbiol Lett 247:91-100

Hijmans RJ, Cameron SE, Parra JL, Jones PG, Jarvis A (2005) Very high resolution interpolated climate surfaces for global land areas. Int $\mathrm{J}$ Climatol 25:1965-1978

Hodkinson ID (2005) Terrestrial insects along elevation gradients: species and community responses to altitude. Biol Rev 80:489-513

Hu HW, Zhang L-M, Dai Y, Di HJ, He JZ (2013) pH-dependent distribution of soil ammonia oxidizers across a large geographical scale as revealed by high-throughput pyrosequencing. J Soils Sediments 13: $1439-1449$

Jumpponen A (2007) Soil fungal communities underneath willow canopies on a primary successional glacier forefront: rDNA sequence results can be affected by primer selection and chimeric data. Microb Ecol 53:233-246

Kitsopoulos KP (1999) Cation-exchange capacity (CEC) of zeolitic volcaniclastic materials: applicability of the ammonium acetate saturation (AMAS) method. Clay Clay Miner 47:688-696

Kuang J-L, Huang L-N, Chen L-X, Hua Z-S, Li S-J et al (2013) Contemporary environmental variation determines microbial diversity patterns in acid mine drainage. ISME J 7:1038-1050
Lauber CL, Strickland MS, Bradford MA, Fierer N (2008) The influence of soil properties on the structure of bacterial and fungal communities across land-use types. Soil Biol Biochem 40:2407-2415

Li J-J, Zheng Y-M, Yan J-X, Li H-J, He J-Z (2013) Succession of plant and soil microbial communities with restoration of abandoned land in the Loess Plateau, China. J Soils Sediments 13:760-769

Lozupone CA, Hamady M, Kelley ST, Knight R (2007) Quantitative and qualitative $\beta$ diversity measures lead to different insights into factors that structure microbial communities. Appl Environ Microbiol 73: $1576-1585$

Luo T, Li W, Zhu H (2002) Estimated biomass and productivity of natural vegetation on the Tibetan Plateau. Ecol Appl 12:980-997

Martiny JBH, Bohannan BJM, Brown JH, Colwell RK, Fuhrman JA et al (2006) Microbial biogeography: putting microorganisms on the map. Nat Rev Microbiol 4:102-112

May LA, Smiley B, Schmidt MG (2001) Comparative denaturing gradient gel electrophoresis analysis of fungal communities associated with whole plant corn silage. Can J Microbiol 47:829-841

McGuire KL, Bent E, Borneman J, Majumder A, Allison SD, Treseder KK (2010) Functional diversity in resource use by fungi. Ecology 91:2324-2332

Mueller RC, Paula FS, Mirza BS, Rodrigues JL, Nüsslein K, Bohannan BJ (2014) Links between plant and fungal communities across a deforestation chronosequence in the Amazon rainforest. ISME J 8: $1548-1550$

Oksanen J, Kindt R, Legendre P, O'Hara B, Stevens MHH, Oksanen MJ, Suggests M (2007) The vegan package: Community ecology package. http://www.R-project.org

Peay KG, Baraloto C, Fine PV (2013) Strong coupling of plant and fungal community structure across western Amazonian rainforests. ISME J $7: 1852-1861$

Pellissier L, Niculita-Hirzel H, Dubuis A, Pagni M, Guex N et al (2014) Soil fungal communities of grasslands are environmentally structured at a regional scale in the Alps. Mol Ecol 23:4274-4290

Pruesse E, Quast C, Knittel K, Fuchs BM, Ludwig W, Peplies J, Glöckner FO (2007) SILVA: a comprehensive online resource for quality checked and aligned ribosomal RNA sequence data compatible with ARB. Nucleic Acids Res 35:7188-7196

Raes J, Bork P (2008) Molecular eco-systems biology: towards an understanding of community function. Nat Rev Microbiol 6:693-699

Rousk J, Bååth E, Brookes PC, Lauber CL, Lozupone C et al (2010) Soil bacterial and fungal communities across a $\mathrm{pH}$ gradient in an arable soil. ISME J 4:1340-1351

Schneider T, Kerblinger KM, Schmid E, Sterflinger-Gleixner K, Ellersdorfer $\mathrm{G}$ et al (2012) Who is who in litter decomposition? Metaproteomics reveals major microbial players and their biogeochemical functions. ISME J 6:1749-1762

Shen C, Liang W, Shi Y, Lin X, Zhang H et al (2014a) Contrasting elevational diversity patterns between eukaryotic soil microbes and plants. Ecology 95:3190-3202

Shen JP, Xu ZH, He JZ (2014b) Frontiers in the microbial processes of ammonia oxidation in soils and sediments. J Soils Sediments 14: 1023-1029

Smit E, Leeflang P, Glandorf B, van Elsas JD, Wernars K (1999) Analysis of fungal diversity in the wheat rhizosphere by sequencing of cloned PCR-amplified genes encoding 18S rRNA and temperature gradient gel electrophoresis. Appl Environ Microbiol 65:2614-2621

Spicer RA, Harris NBW, Widdowson M, Herman AB, Guo S et al (2003) Constant elevation of southern Tibet over the past 15 million years. Nature 421:622-624

Sundqvist MK, Sanders NJ, Wardle DA (2013) Community and ecosystem responses to elevational gradients: processes, mechanisms, and insights for global change. Annu Rev Ecol Evol Syst 44:261-280

Wang JJ, Soininen J, He JZ, Shen J (2012) Phylogenetic clustering increases with elevation for microbes. Environ Microbiol Rep $4: 217-226$ 
Wang J-T, Cao P, Hu H-W, Li J, Han L-L et al (2014) Altitudinal Distribution patterns of soil bacterial and archaeal communities along Mt. Shegyla on the Tibetan Plateau. Microb Ecol 69:135-145

Yang Y, Gao Y, Wang S, Xu D, Yu H et al (2013) The microbial gene diversity along an elevation gradient of the Tibetan grassland. ISME J 8:430-440
Zhang LM, Wang M, Prosser JI, Zheng YM, He JZ (2009) Altitude ammonia-oxidizing bacteria and archaea in soils of Mount Everest. FEMS Microbiol Ecol 70:208-217

Zhang XF, Zhao L, Xu SJ, Liu YZ, Liu HY, Cheng GD (2013) Soil moisture effect on bacterial and fungal community in Beilu River (Tibetan Plateau) permafrost soils with different vegetation types. J Appl Microbiol 114:1054-1065 